\title{
PREFERÊNCIA DE HABITAT DE OLIGOCHAETA(ANNELIDA, CLITELLATA) EM MACRÓFITAS AQUÁTICAS NA REPRESA RIBEIRÃO DAS ANHUMAS (AMÉRICO BRASILIENSE-SP)
}

SANCHES, Nathalie Aparecida de Oliveira. Graduação em Ciências Biológicas - Centro Universitário de Araraquara - Uniara. E-mail: nathalie_sanches@hotmail.com.

GORNI, Guilherme Rossi. Mestrado em Desenvolvimento Regional e Meio Ambiente - Departamento de Ciências Biológicas e da Saúde - Centro Universitário de Araraquara - Uniara. E-mail: grgorni@ gmail.com.

\section{Resumo}

As macrófitas possuem complexidades estruturais morfológicas diferentes, oferecendo aos animais a disponibilidade de vários nichos. Esses vegetais também são um importante substrato para o desenvolvimento de perifíton, que possui grande valor nutricional e constitui um dos principais alimentos dos invertebrados aquáticos, principalmente naidídeos. Este estudo teve como objetivo verificar a diversidade da comunidade de Oligochaeta em macrófitas dos gêneros Egeria sp. e Salvinia sp., em lagoas marginais da represa do Ribeirão das Anhumas. Essas macrófitas possuem características tridimensionais distintas e hábitos diferentes, sendo a Egeria submersa fixa e a Salvinia flutuante livre. As coletas das macrófitas foram realizadas entre os meses de agosto de 2012 e abril de 2013. Das amostras vegetais foram tomadas 100 gramas de biomassa (peso úmido) de cada gênero e a remoção das plantas do ambiente foi feita com o auxílio de uma peneira com malha $0,21 \mathrm{~mm}$. Entre as duas macrófitas analisadas, a Egeria sp. foi a que obteve maior diversidade, riqueza e abundância em relação à Salvinia sp. Esses resultados demonstram que as macrófitas aquáticas são importantes para o estabelecimento dos oligoquetos, fornecendo principalmente proteção e alimento, e que possivelmente a morfologia e hábito das plantas são os fatores que mais influenciam na associação da oligogauna com esses vegetais.

Palavras-chave: Oligoquetos aquáticos; Substrato vegetal; Plantas aquáticas.

Oligochaeta (Annelida, Clitellata) in the aquatic macrophytes in dam of Ribeirão Of Anhumas SCreamers (AMÉrico BrasilienSE-SP)

\begin{abstract}
Macrophytes have different morphological structural complexities, offering to animals the availability of various niches. These plants are also an important substrate for the development of periphyton, which has ahigh nutritional value and is one of the main foods of aquatic invertebrates, mainly Naididae. This study aimed at examinining the diversity of Oligochaeta community in macrophytes belonging to genus Egeria sp. and Salvinia sp., in lagoons of Ribeirão das Anhumas dam. These macrophytes have distinct three-dimensional characteristics and different habits, being Egeria fixed submerged and Salvinia free floating. The collections of macrophytes were carried out between the months of August 2012 and April 2013. Samples of 100g (wet weight) of each genus were taken from plant biomass and the removal of the plants from the environment was made with the aid of a sieve with $0.21 \mathrm{~mm}$ mesh. Considering the two macrophytes analyzed, Egeria sp. was the one that presented greater diversity, richness and abundance in relation to Salvinia sp. These results demonstrate that macrophytes are important for the establishment of oligochaetes, mainly providing protection and food, and possiblythe morphology and habit of the plants are the most influential factors in the association of oligofauna with these plants.
\end{abstract}

KEYwORDs: Aquatic oligochaetes; Vegetable substrate; Aquatic plants. 


\section{INTRODUÇão}

Segundo Esteves (1998), na região litorânea de ecossistemas aquáticos continentais podem ser encontradas diferentes comunidades de macrófitas. Esses vegetais influenciam todo o ecossistema lacustre e propicia melhores condições para o estabelecimento dos oligoquetos e da fauna bentônica em geral. As macrófitas possuem complexidades estruturais morfológicas diferentes, oferecendo aos animais a disponibilidade de vários nichos (ROSINE, 1955). As macrófitas também são um importante substrato para o desenvolvimento de perifiton, que possui grande valor nutricional e constitui um dos principais alimentos dos invertebrados aquáticos (POTT e POTT, 2000), principalmente espécies de Naididae (LEARNER e LOCHHEAD, 1978), o que, possivelmente, é um dos fatores que mais influenciam a associação desses organismos a esses vegetais, tornando as populações dessa família um grupo frequentemente dominante perante os outros oligoquetos em lagoas com densa vegetação (Brinkhurst e Jamieson, 1971). Ademais, plantas aquáticas podem ocupar locais distintos na coluna d'água, sendo classificadas, segundo Pott e Pott (2000), como: macrófitas anfíbias, emergentes, flutuantes fixas, flutuantes livres, submersas fixas, submersas livres e epífitas. As características do sedimento, disponibilidade de oxigênio, morfologia das margens, presença de vegetação, estágio de decomposição das folhas (SMOCK e STONEBURNER, 1980), profundidade, natureza química do substrato e disponibilidade de alimentos são fatores que exercem forte influência sobre a distribuição e abundância da comunidade bentônica (LEARNER e LOCHHEAD, 1978; CORBI, 2001).

Diversos autores já registraram oligoquetos vivendo associados a plantas aquáticas (CORREIA e TRIVINHO-STRIXINO, 1998; ALVES e GORNI, 2007; CORBI e TRIVINHO-STRIXINO, 2002), entretanto, ainda são encontradas lacunas no conhecimento sobre aspectos ecológicos dessas comunidades associadas a substratos vivos.

Assim, este estudo teve como objetivo verificar a diversidade da comunidade de Oligochaeta, em macrófitas dos gêneros Egeria sp. e Salvinia sp., em lagoas marginais da represa do Ribeirão das Anhumas.

\section{Metodologia}

\section{Local de Estudo}

O presente estudo foi realizado em lagoas marginais da represa do Ribeirão das Anhumas. Essa represa está localizada no Município de Américo Brasiliense, região central do Estado de São Paulo, nas coordenadas geográficas $21^{\circ} 42^{\prime 2} 23^{\prime \prime}$ latitude Sul e $48^{\circ} 0$ '33" longitude Oeste a $544 \mathrm{~m}$ de altitude (Figura 1). Possui um sedimento arenoso e uma profundidade média entre 3,70m e 7,60m, distribuída em uma área total de $0,82 \mathrm{Km}^{2}$. Ao redor há uma área florestada, além de uma vasta vegetação de macrófitas localizadas à montante da represa (CORBI e TRIVINHOSTRIXINO, 2002; PEIRÓ e ALVES, 2006). 


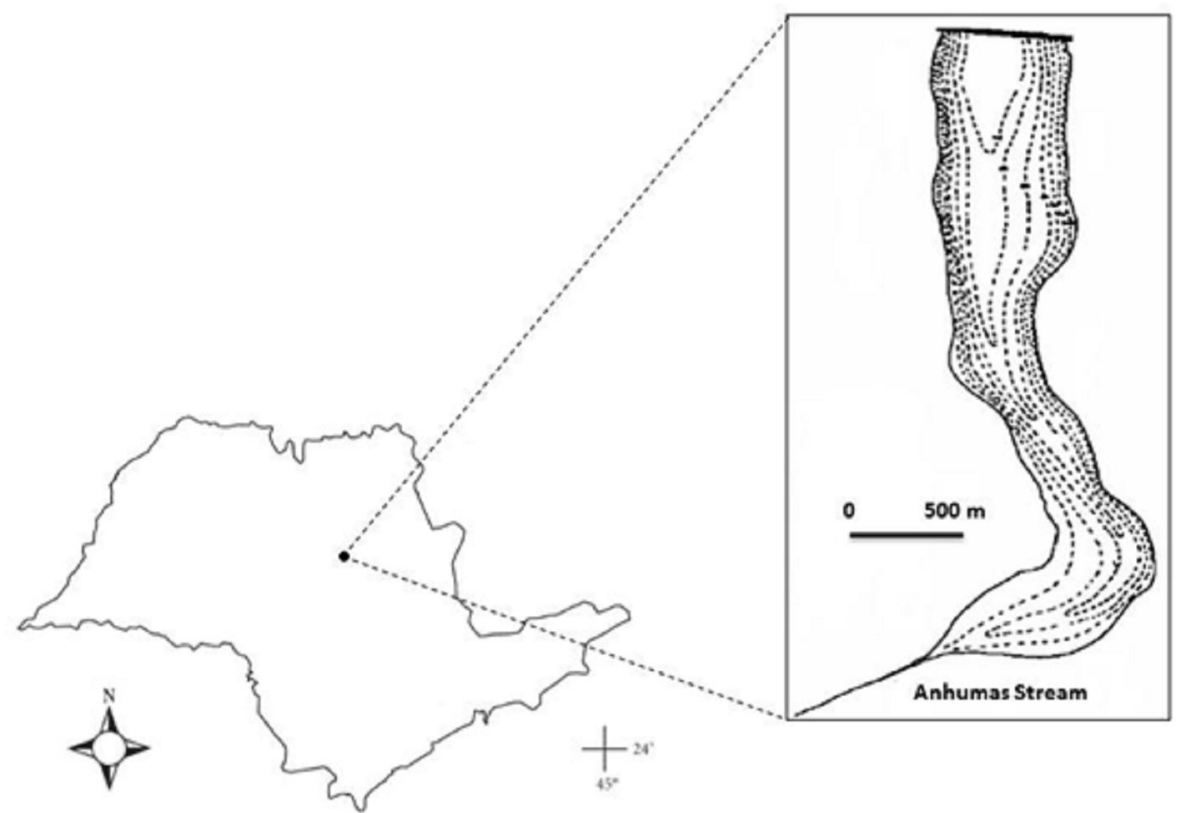

Figura 1 - Mapa batimétrico da Represa Ribeirão das Anhumas e sua localização geográfica no estado de São Paulo.

Fonte: Adaptado de Corbi (2001).

\section{Coleta e identificação}

As coletas foram realizadas em intervalos trimestrais durante o período de agosto de 2012 e abril de 2013 na região litorânea das lagoas.
As macrófitas coletadas foram identificadas, segundo Pott e Pott (2000), e pertencem aos gêneros Egeria (Hydrocharitaceae) e Salvinia (Salvinaceae) (Figura 2).

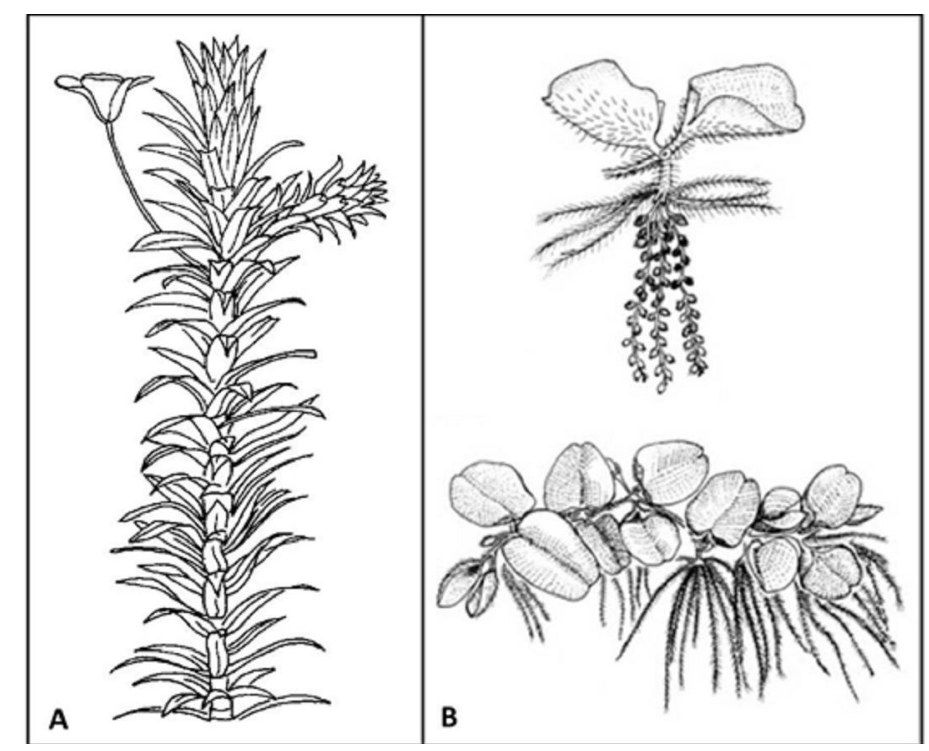

Figura 2 - Visão geral de um espécime de Egeria sp. (A) e Salvinia sp. (B).

Fonte: Dos autores. 
As amostras vegetais foram acondicionadas em galões plásticos contendo água do próprio ambiente, e conduzidas ao laboratório. Logo após, realizou-se uma lavagem das macrófitas sobre peneira granulométrica com malha de $0,21 \mathrm{~mm}$, visando ao desprendimento e seleção dos organismos, que foram triados, fixados com formalina $10 \%$ e, posteriormente, conservados emálcool 70\% (PEIRÓ e ALVES, 2006; ALVES e GORNI, 2007; PEIRÓ e GORNI, 2010).

Para identificação das espécies de oligoquetos, foram montadas lâminas semipermanentes e adotados critérios taxonômicos propostos por Brinkhurst e Jamieson (1971), Righi (1984), Brinkhurst e Marchese (1989).

\section{Análises dos dados}

Visando obter uma relação de dispersão da composição da fauna em relação às diferentes condições do substrato (macrófitas), foi obtido um diagrama de ordenação resultante de um escalonamento multidimensional não métrico (n-MDS) com índice de Morisita. Para essa análise foram utilizados dados logaritimizados de abundância de todas as coletas correspondentes às macrófitas aquáticas Egeria sp. e Salvinia sp. Esses dois gêneros foram escolhidos devido às diferenças marcantes na complexidade tridimensional oferecida pelas características morfológicas da planta e seus hábitos distintos, ocupando locais diferentes na coluna d' água. A macrófita Egeria sp. é submersa fixa, enraizada no fundo, seu caule e folhas ficam submersos, geralmente saindo somente suas flores para fora da água, além de ser macia ao tato, frágil e conter muito perífiton aderido a suas estruturas. Já Salvinia sp. é uma planta flutuante livre, não enraizada no fundo, com caule, ramos e/ou folhas flutuantes. Suas folhas possuem pêlos que repelem a água, ao contrário de suas raízes, que são folhas modificadas, e seguramágua e sedimentos como uma esponja (POTT e POTT, 2000).

Para a realização das análises foiutilizado o software Palaeontological Statistics (PAST - versão 1.49) (HAMMER et al., 2001).

\section{Resultados}

Foram inventariadas 18 espécies (de um total de 395 organismos), distribuídas em duas famílias: Família Naididae (Subfamília Naidinae, Subfamília Pristininae e Subfamília Tubificinae) e Família Opistocystidae. Naididae representou $87,8 \%$ da amostra e foi composta por oito gêneros: Allonais, Aulophorus, Chaetogaster, Dero, Pristina, Slavina, Stylaria, Aulodrilus, dos quais o gênero Pristina apresentou maior abundância, 24,8\%. A família Opistocystidae foi representada por uma única espécie, Opistocysta funiculus, que representou $12,2 \%$ da amostra total de organismos (Tabela 1). 
Tabela 1 - Espécies de Oligochaeta associadas às macrófitas aquáticas Egeria sp. e Salvinia sp. em lagoas adjacentes a Represa Ribeirão das Anhumas. \%: ocorrência da espécie no total amostrado.

\begin{tabular}{lccc}
\hline Es pécies de Oligochaeta & Egeria & Salvinia & Total \\
\hline Allonais paraguayensis paraguayensis (Michaelsen, 1905) & - & 2 & 2 \\
Aulophorus costatus Du Bois-Reymond Marcus, 1944 & 2 & 45 & 47 \\
Aulophorus borellii (Michaelsen, 1900) & 1 & 1 & 2 \\
Chaetogaster diaphanus (Gruithuisen, 1828) & 4 & 1 & 5 \\
Dero digitata (O.F. Müller, 1773) & 5 & 5 & 10 \\
Dero nivea Aiyer, 1930 & 33 & 15 & 48 \\
Dero sawayai Marcus, 1943 & 2 & - & 2 \\
Slavina evelinae (Marcus, 1942) & 18 & 3 & 21 \\
Stylaria lacustris (Linnaeus, 1767) & 77 & 3 & 80 \\
Pristina biserrata Chen, 1940 & 5 & 1 & 6 \\
Pristina breviseta Bourne, 1891 & 1 & - & 1 \\
Pristina longiseta Ehrenberg, 1828 & 7 & 61 & 68 \\
Pristina macrochaeta Stephenson, 1931 & 2 & - & 2 \\
Pristina proboscidea Beddard, 1896 & 1 & 1 & 2 \\
Pristina rosea (Piguet, 1906) & 3 & - & 3 \\
Pristina synclites Stephenson, 1925 & 16 & - & 16 \\
Aulodrilus pigueti Kowalewski, 1914 & 32 & - & 32 \\
Opistocystafuniculus Cordero, 1948 & 48 & - & 48 \\
\hline Total & 257 & 138 & 395 \\
\hline
\end{tabular}

Fonte: Dados de pesquisa.

Entre as duas macrófitas analisadas, a Egeria sp. obteve valores de diversidade, riqueza e abundância maiores que a espécie Salvinia sp. (Tabela 2). A análise n-MDS demonstrou que há variação da comunidade de Oligochaeta em relação aos substratos vegetais analisados, visto que foi observada nítida separação, no eixo das abiscissas, entre a fauna associada às macrófitas Egeria sp. e Salvinia sp. (Figura 3). 
Tabela 2 - Valores de riqueza, abundância, Índice de diversidade de Shannon $\left(\mathrm{H}^{\prime}\right)$ e equitabilidade das macrófitas Egeria sp. e Salvinia sp.

\begin{tabular}{lcc}
\hline & Egeria $s p$. & Salvinia sp. \\
\hline Riqueza & 18 & 11 \\
Abundância & 257 & 138 \\
$\mathrm{H}^{\prime}$ & 2,12 & 1,458 \\
Equitabilidade & 0,7335 & 0,6082 \\
\hline
\end{tabular}

Fonte: Dados de pesquisa.

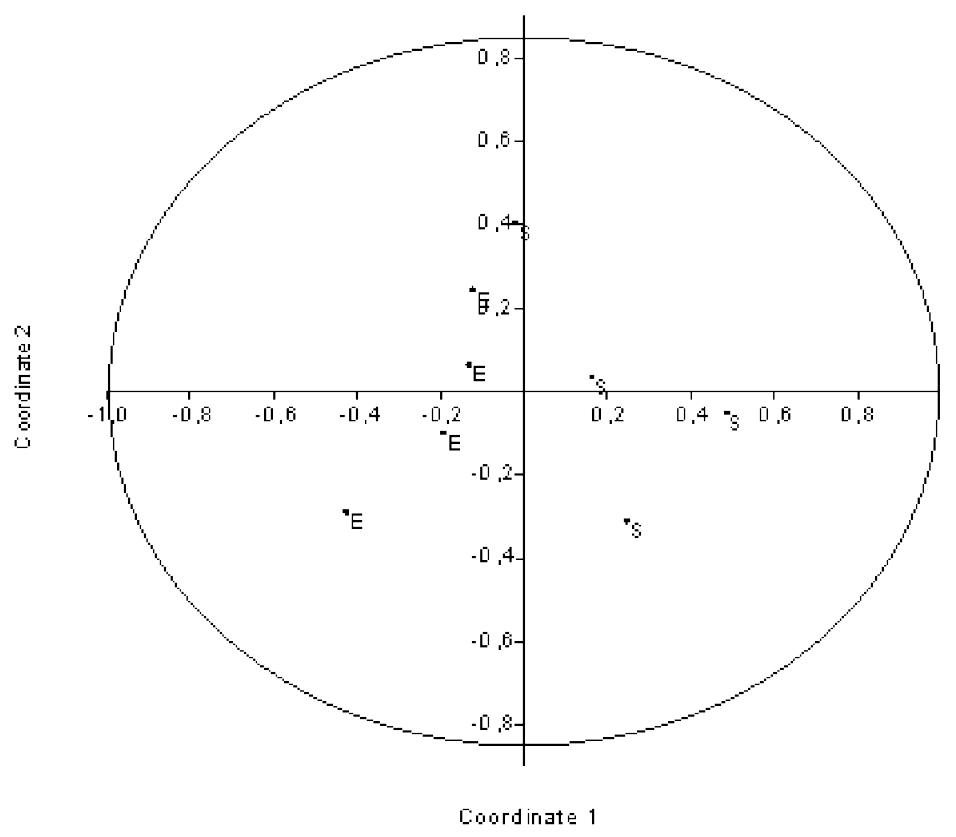

Figura 3 - n-MDS das amostras de macrófitas coletadas nas lagoas anexas ao Ribeirão das Anhumas, Américo Brasiliense-SP. E: Egeria sp.; S: Salvinia sp. Valor do stress = 0,115.

Fonte: Dados de pesquisa.

\section{Discussão}

Neste estudo, o elevado registro de espécies de Naididae indica que esta família encontra nas macrófitas nichos apropriados para seu estabelecimento e sobrevivência. Vários autores já citaram que o desenvolvimento de Naididae é favorecido pela vegetação aquática (LEARNER e LOCHHEAD, 1978; CÓ, 1979; CORBI e TRIVINHO-STRIXINO, 2002; ALVES e GORNI, 2007). Esse fato provavelmente está ligado ao acúmulo de perifíton na superfície e fendas das estruturas morfológicas vegetais, principalmente folhas e raízes.

Os estudos de Brinkhurst e Jamieson (1971) mostraram que o perifíton, juntamente aos detritos acumulados nas plantas, são a maior fonte nutricional para naidídeos, o que pode explicar a elevada abundância dessa família junto à Egeria sp. neste trabalho, uma vez que a morfologia desse vegetal parece fornecer características favoráveis à oligofauna por apresentar pequenas folhas macias cheias de perifíton 
e sedimento aderido, além de possuir hábito submerso, ocupando profundidades maiores na coluna d'água (POTT e POTT, 2000).

Alves e Gorni (2007) coletaram Egeria sp. na Represa Ribeirão das Anhumas, e registraram seis espécies associadas, sendo elas: Dero digitata, Dero nivea, Dero sawayai, Dero raviensis, Pristina longiseta e Stylaria lacustris. Foi uma riqueza menor em comparação com este trabalho, que obteve uma riqueza de 18 espécies. Das espécies registradas pelos autores, $D$. nivea e $S$. lacustris foram as mais abundantes. Similarmente, neste estudo, essas duas espécies foram bastante significativas, juntamente com Pristina longiseta. Em pesquisa similar, Corbi e Trivinho Strixino (2002) identificaram dez espécies em regiões da represa com macrófitas aquáticas, sendo oito delas da família Naididae, das quais Allonais chelata, Allonais paraguayensis, Dero nivea, Pristina breviseta, Slavina evelinae e Opystocysta funiculus também foram registradas no presente trabalho.

A riqueza de oligoquetos coletados junto à Salvinia sp. foi menor, mas ainda assim a planta obteve muitos indivíduos associados principalmente a suas raízes. De acordo com Heckman (1998), os vegetais flutuantes desenvolvem um sistema radicular denso, que fornece um biótopo favorável para o abrigo de diversos organismos aquáticos. Neste estudo a espécie Aulophorus costatus foi muito representativa no verão, sendo encontrada dentro de tubículos feitos com partículas de raízes de Salvinia. De acordo com Pott e Pott (2000), as raízes dessa macrófita são na verdade folhas modificadas, que atuam como esponjas, retendo água e grande quantidade de sedimento. Essa retenção de matéria orgânica e sedimento aparentemente é o motivo da ocorrência da oligofauna exclusivamente nas raízes dessa planta, na qual os organismos encontram alimento e matéria-prima para a construção dos tubículos. Similarmente, Armendáriz (2008) registrou A. costatus associada à macrófita flutuante Azolla sp. (Azollaceae), caracterizando uma possível preferência desse oligoqueto por plantas com esse hábito, que, segundo a autora, pode estar relacionado ao desenvolvimento de bactérias e algas perifíticas na superfície do vegetal. Trivinho-Strixino et al., (2000), identificaram quatro espécies de oligoquetos com baixas densidades associados à Salvinia auriculata: Allonais paraguayensis, Dero nivea, Dero evelinae e Aulophorus lodeni. Além desses trabalhos, Salcedo (2011) encontrou grande número de oligoquetos em Salvinia auriculata, porém não realizou a identificação até nível de espécie.

A única espécie pertencente a outra família foi Opistocysta funiculus, que aparentemente ocupa nichos muitos parecidos aos Naididae, principalmente por apresentar algumas semelhanças morfofisiológicas com essa família, tanto que já foi classificada como Pristina flagellum (Cernosvitov, 1936), devido à presença de probóscide. Além disso, ela possui três apêndices caudais, parecidos com os do gênero Aulophorus (MARCUS, 1944). Corbi e TrivinhoStrixino (2002) encontram essa espécie com participação maior que $10 \%$ nas áreas com densa vegetação aquática, areia fina e grande quantidade de matéria orgânica, na represa Ribeirão das Anhumas.

\section{CONSIDERaÇões Finais}

Macrófitas aquáticas demonstraram ser um importante substrato para a oligofauna e, aparentemente, sua presença é um dos fatores que influenciam a distribuição da comunidade local. Dentre as macrófitas estudadas, Egeria sp. foi o substrato mais utilizado pelos oligoquetos, possivelmente devido a sua alta complexidade tridimensional e seu hábito submerso, o que acarreta o aumento do perifíton aderido em suas estruturas.

Os resultados obtidos no presente estudo vêm contribuir de forma significativa para o conhecimento dos oligoquetos aquáticos, principalmente características de sua ecologia, destacando sua forte relação com as macrófitas aquáticas. Entretanto, esses dados não são conclusivos, e sugerem novos estudos para ampliar as informações e importância desses organismos na dinâmica dos ecossistemas aquáticos continentais brasileiros. 


\section{REFERÊNCIAS}

ALVES, R.G.;GORNI, G. R. Naididae species (Oligochaeta) associated with submersed aquatic macrophytes in two reservoirs. São Paulo. Acta Limnologica Brasiliensia, v.19, n.4, p.407-413, 2007.

ARMENDÁRIZ, L.C. Ciclo de Vida de Dero (Aulophorus) costatus Marcus, 1944 (Tubificidae, Oligochaeta) en un cuerpo de agua com vegetation flotante en los Talas, Argentina. Gayana, v.72, n.1, p. 23-20, 2008.

BRINKHURST, R.O; JAMIESON, B.G.M. Aquatic Oligochaeta of the world. University of Toronto Press. 1971, 860p.

BRINKURST, R.O.; MARCHESE, M.R. 1989. Guia para la identificación de oligoquetos acuáticos continentales de Sud y

Centroamerica. Santa Fé: Asociación de ciencias naturales del litoral, 1989. 207p.

\section{CÓ, L. M. Distribuição de oligochaeta na}

Represa do Lobo (Estado de São Paulo, Brasil). 1979. 169 f. Mestrado (Limnologia) - Centro de Ciências Biológicas e da Saúde, Universidade Federal de São Carlos, São Carlos, São Paulo.

\section{CÓ, L. M. Distribuição de oligochaeta na}

Represa do Lobo (Estado de São Paulo, Brasil). 1979. 169 f.. Mestrado (Limnologia) - Centro de Ciências Biológicas e da Saúde, Universidade Federal de São Carlos, São Carlos.

\section{CORBI, J. J. Distribuição espacial e batimétrica dos macroinvertebrados bentônicos da Represa do Ribeirão das Anhumas (Américo Brasiliense- SP). 2001.75 f. Dissertação (Mestrado em Ecologia e Recursos Naturais) - Centro de Ciências Biológicas e da Saúde, Universidade Federal de São Carlos, São Carlos, São Paulo.}

CORBI, J. J.; TRIVINHO-STRIXINO, S. Spatial and bathymetric distribution macrobenthic fauna of the Ribeirão das Anhumas reservoir (Américo Brasiliense-SP, Brasil). Acta Limnologica Brasiliensia, v.10, n.1, p. 37-47, 2002.

CORREIA, L.C.S.; TRIVINHO-STRIXINO, S Macroinvertebrados da Rizosfera de Scirpus cubensis na lagoa do Infernão (Estação Ecológica de Jataí-SP): Estrutura e função. Acta Limnologica Brasiliensia, v.10, n.1, p. 37-47, 1998.

ESTEVES, F.A. Fundamentos de limnologia. 2 ed. Rio de Janeiro: Interciência, 1998. 602 p.

HAMMER, Ø.; HARPER, D. A.T.; RYAN P. D. Past: Paleontological Statistics Software Package for Education and Data Analysis. Palaeontologia Electronica, E.U.A, v. 4, n. 1, p. 1-9, 2001.

HECKMAN, C.W. The Seasonal Succession of Biotic Communities in Wetlands of the Tropical Wetand-Dry Climatic Zone: V. Aquatic Invertebrate Communities in the Pantanal of Mato Grosso, Brazil. International Rewiew of Hydrobiology, v.83, n. 1, p. 31-63.

LEARNER, M.A; LOCHHEAD, G;HUGHES, B.D. Areview of the biology of the Britishi Naididae (Oligochaeta) with emphasis on the lotic environment. Freswater biology, v.8, p. 357-375, 1978.

MARCUS, E. Sobre Oligochaeta límnicos do Brasil. Boletim da Faculdade de Filosofia, Ciências e Letras da Universidade de São Paulo Zoologia.v.43, n.8, p.5-135, 1944.

PEIRÓ, D.F.; ALVES, R.G. Insetos aquáticos associados a macrófitas da região litoral da represa do Ribeirão das Anhumas(Município de Américo Brasiliense, São Paulo, Brasil). Biota Neotropica, v. 6, n. 2, p. 1-9, 2006. 
PEIRÓ, D.F; GORNI, G.R. Diferença na composição da entomofauna aquática associada a duas plantas de hábitos diferentes em um tanque de psicultura. Multiciência, v.10, p. 149-160, 2010.

POTT, V.J.; POTT, A. Plantas aquáticas do

Pantanal. Embrapa, 2000.353 p

RIGHI, G. 1984. Manual de identificação de invertebrados límnicos do Brasil. CNpq/

Coordenação Editorial. 48p.

ROSINE, W.N. The Distribution of invertebrates on submerged aquatic plant surfaces in Muskee Lake, Colorado. Ecology, v. 36, n. 2, p.308-314, 1955.
SALCEDO, A.K.M. Riqueza e densidade de macroinvertebrados aquáticos associados às macrófitas aquáticas nos lagos de várzea Januacá (AM) e Grande de Curuaí (PA) da Amazônia Central Brasileira. 2011. 102 p. Tese de Doutorado (Programa de Pós-Graduação em Ecologia) - Instituto de Ciências Biológicas Departamento de Ecologia - Universidade de Brasília. Brasília, Distrito Federal.

TRIVINHO-STRIXINO, S.; CORREIA, L.C.S.; SONODA, K. Phytophilous Chironomidae (Diptera) and other macroinvertebrates in the oxbow Infernão Lake (Jataí Ecological Station, Luiz Antônio, SP, Brazil). Revista Brasileira de Biologia, v.60, n.3, p.527-535, 2000. 